CINAHL, BIOSIS, Cochrane, ISI Web of Science, Lilacs, Campbell and SCEH.

Results In total, 48740 records were identified. 95 empirical studies were identified as relevant and included in the analysis. The majority of adapted intervention studies took place in the USA, conducted with African-Caribbean origin populations and these predominantly involved women. All studies conducted with Chinese-origin populations took place in the USA while the majority of studies with South Asian-origin populations were conducted in the UK. Multi-component interventions targeting physical activity and nutrition were the most common followed by smoking cessation interventions. Interventions utilised a variety of adapted methods, resources and/or settings. The components of the adaptation process identified include methods such as ethnically matching programme facilitators; subsidising gym memberships and promoting low-cost alternatives to usual exercise options. Resources include culturally targeting materials (eg, using ethnic actors in videos and including photos of foods commonly consumed by the population in promotional material); utilising existing community resources (eg, religious leaders) and accommodating for differing linguistic and language competencies. Settings include holding interventions in familiar locations and utilising culturally appropriate scenarios to elicit behaviour change.

Conclusions A large body of evidence exists for adapted interventions. Identification of the components involved in the adaptation process for ethnic minority populations is a critical step for building on existing adaptation principles. Furthermore, this study will enable the development of a framework to guide the adaptation of mainstream evidence-based guidelines to be salient for different populations and contexts.

\section{P50 "PUSHING AGAINST THE HILL": A QUALITATIVE STUDY OF IRISH TRAVELLER HEALTH IN THE 21ST CENTURY}

doi:10.1136/jech.2010.120477.50

J Turner, R NicChárthaigh, B Quirke, J Kilroe, N A Hamid, C Kelly, C C Kelleher, R G Moore, for the All Ireland Traveller Health Study group. UCD, Dublin, Ireland

Background Travellers are a distinct minority grouping, characterised by a nomadic tradition and shared cultural traditions, who experience poor health and social disadvantage.

Objective To explore in-depth a series of important issues related to the social determinants of health with members of the Irish Traveller community.

Setting This paper presents data from 26 focus groups conducted with the Traveller community in the Republic of Ireland (ROI) and Northern Ireland (NI). The data presented are a sub-study of the AllIreland Traveller Health Study. The focus groups incorporated a geographical spread, a gendered and age-related perspective and compared and contrasted the findings across ROI and NI.

Methodology Participatory methodology ensured that the Traveller community was consulted throughout the research progress and ingress was achieved. The focus groups were recruited via the Traveller Health Network. Peer researchers were co-trained by Pavee Point, the Traveller stakeholder organisation and by university research staff to act as co-facilitators and mediators. Focus groups were transcribed and thematically analysed using grounded theory and the constant comparison method and were validated by inter-raters.

Results The analysis produced rich data which reached saturation. Key thematic issues arose from the data. These non-comprehensively included rapid historical changes in the economic, cultural and policy arenas have impacted on the traditional lived experience of Travellers; nomadism has decreased whilst accommodation problems have increased marginalisation and isolation; a recognised sense of loss of Traveller culture, sense of identity and self esteem but a resilience and determination to seek new forms of meaning; new cultural conditions impacting directly on the quality of life and health chances influencing social and institutional opportunities and barriers; High rates of discrimination perceived in NI and ROI; Irish Traveller community is not a homogenous community; major concerns regarding the increase in drug culture in ROI, although less marked in NI; education as a continuing source of concern during the educational process and beyond; new mass communication technology embraced by some (younger/literate) Travellers as an important source of information, exchange and sociability.

Conclusion The findings echo and reinforce previous evidence but also highlight novel issues. Based on their own account, Travellers continue to face multiple health challenges that impact directly upon their physical and mental health. It is a time of flux and disembodying mechanisms in the broader culture that can generate both positive and negative developments. Travellers have interpreted these experiences as "pushing against a hill".

\section{Health services/Policy

P51 SURVIVING INTENSIVE CARE: A SYSTEMATIC REVIEW OF
HEALTH CARE RESOURCE USE AFTER HOSPITAL
DISCHARGE

doi:10.1136/jech.2010.120477.51

${ }^{1} \mathrm{~N}$ I Lone, ${ }^{1} \mathrm{M}$ Seretny, ${ }^{2} \mathrm{~K}$ M Rowan, ${ }^{3} \mathrm{~T}$ S Walsh, ${ }^{1} \mathrm{~S}$ H Wild, ${ }^{1} \mathrm{G}$ D Murray. ${ }^{1}$ Centre for Population Health Sciences, University of Edinburgh, Edinburgh, UK; ${ }^{2}$ Intensive Care National Audit and Research Centre, London, UK, ${ }^{3}$ Department of Anaesthesia, Critical Care and Pain Medicine, University of Edinburgh, Edinburgh, UK

Background Intensive care units (ICUs) are an expensive resource. However, this expense does not end at hospital discharge. ICU survivors continue to experience significant morbidity. As the demand for ICU is likely to increase substantially, there is a need to establish how much health care resource survivors consume following discharge from hospital. This will enable appropriate service planning and policy development to meet the needs of these patients, and will improve the precision of economic evaluations relating to ICU.

Aims We conducted a systematic review to determine the reported use of major health care resource by ICU survivors following discharge from hospital and to identify factors associated with increased resource use.

Methods Studies were included if the study population derived from an adult, general ICU population, health care resource use was reported at the patient level and the publication was in the English language. Two reviewers independently screened abstracts, rejecting those clearly not meeting inclusion criteria. A single reviewer then retrieved the full texts and assessed them for inclusion. Costs were inflated to 2009 using the consumer price index and converted to US dollars using the purchasing power parity method.

Results From 3522 articles, nine fulfilled criteria for inclusion. Two studies were conducted in the UK; three in Canada and four in the USA. Six studies used a cohort design; the remaining three collected data as part of a trial. The number of patients for which resource use was reported ranged from 66 to 963. Mean age ranged from 40 to 66. There was substantial variation in the cost categories included in each study. Following standardisation to a common currency and year, variation in resource use was apparent (range \$1610-\$45173). Studies undertaken within the USA reported the highest costs; those in the UK reported substantially lower costs. The larger proportion of resource was consumed in secondary care (range $53-96 \%)$. Factors associated with increased resource use included increasing age, co-morbidities and organ dysfunction score.

Conclusion This review is the first to bring together the literature relating to post-hospital discharge health care resource use for survivors of ICU. There was substantial variation in the cost of resource use between studies. Given the paucity of identified studies and their 
relatively short time horizons, there is a clear need for longer term studies to investigate resource use of ICU survivors. Our findings should help to inform the design and reporting of such studies.

\section{P52 PREDICTORS OF EMERGENCY DEPARTMENT ATTENDANCE RATES IN SMALL AREA POPULATIONS}

doi:10.1136/jech.2010.120477.52

${ }^{1} \mathrm{G}$ Rudge, ${ }^{1} \mathrm{~S}$ Fillingham, ${ }^{2} \mathrm{~K}$ Sidhu, ${ }^{1} \mathrm{M}$ Mohammed. ${ }^{1}$ School of Health and Population Sciences, The University of Birmingham, Birmingham, UK; ${ }^{2}$ West Midlands Strategic Health Authority, Birmingham, UK

Objectives Emergency Department (ED) use is known to be related to both socio-economic deprivation and distance to hospital. However, hospitals are often located in urban areas close to deprived populations. The objective of this study is to investigate the relationship between distance and attendance at EDs, net of deprivation. Method Multiple linear regression models were used to explore the relationship between deprivation and distance to hospital with agesex standardised ED attendance rates at neighbourhood level. Distance to a Minor Injury Unit (MIU) was also included. Interaction between distance and deprivation was investigated. Separate models for children and adults were used because some populations had access to a dedicated children's ED.

Setting The Government Office Region of the West Midlands, in central England (population 5.4 million).

Population Attendances of West Midlands residents to EDs in acute hospitals in financial year 2007/08 $(n=1465355)$ within 3482 Lower Level Super Output Areas (LSOA), each containing approximately 1600 residents.

Outcome Measures The response variable was the directly age-sex standardised ED attendance rate for each LSOA (square-root transformed). Predictor variables were distance from LSOA centroid to nearest ED and nearest MIU in kilometres and income deprivation score (high score=high deprivation).

Results There was a significant positive relationship between attendance and deprivation, $\beta$ coefficient $=0.96(p<0.001)$ in adults and $0.68(p<0.001)$ in children. There was a significant negative relationship between attendance and distance, the $\beta$ coefficient $=-0.24(p<0.001)$ in adults and $-0.37(p<0.001)$ in children. Child attendance appeared more sensitive to distance than adult attendance but less sensitive to deprivation. Attendance in deprived neighbourhoods was more sensitive to distance than in less deprived ones. For adults, at the third quartile of deprivation, $\sqrt{ }$ attendance rate $=-0.32$ per $\mathrm{km}+19.50$ compared to -0.18 per $\mathrm{km}+15.86$ at the fist quartile. For children, at the third quartile of deprivation, $\sqrt{ }$ attendance rate $=-0.49$ per $\mathrm{km}+21.30$ compared to -0.30 per $\mathrm{km}$ +17.95 at the first quartile. The paper goes on to describe the models in greater detail, (including the effect of MIUs).

Conclusion Attendance at EDs is sensitive to income deprivation at neighbourhood level but distance from hospital appears to modify this relationship differently in deprived areas than in affluent ones. Also, children's attendance appears to be more sensitive to distance than that of adults. This is useful to understand when comparing ED attendance between and within populations.

\section{P53 MORTALITY AFTER HOSPITAL ADMISSION CLEARLY VARIES BETWEEN ATHEROSCLEROTIC DISEASES LOCATED AT DIFFERENT VASCULAR BEDS}

doi:10.1136/jech.2010.120477.53

I Vaartjes, D E Grobbee, Bots. Julius Centre for Health Sciences and Primary Care, University Medical Centre Utrecht, Utrecht, The Netherlands

Objective Most studies providing data on survival in patients with atherosclerosis only address a single disease site: heart, brain or legs.
Therefore, our objective was to determine risk of death after first hospital admission for atherosclerotic disease located at different sites.

Design Hospital-based follow-up study.

Setting and Participants A nationwide cohort of patients hospitalised for the first time for acute myocardial infarction (AMI), peripheral arterial disease of the lower extremities (PAD) or ischaemic stroke was identified through linkage of national registers in 1995, 1997 and 2000. The total population of the Netherlands in 1995, 1997 and 2000 was 15424122 (men 7627 428, women 7 796640), 15567107 (men 7696803 , women 7870304 ) and 15863950 (men: 7846317 , women: 8017633$)$, respectively.

Main Outcome Measure Twenty-eight-day, 1-year and 5-year mortality rate in AMI patients was compared to mortality rate in ischaemic stroke patients and $\mathrm{PAD}$ patients by estimating RR (with $95 \% \mathrm{CI}$ ). Cox's proportional hazard models were used to adjust for age.

Results Case fatality was highest for ischaemic stroke patients though lowest for PAD patients. In patients 55 to 74 years, large differences in risk of death were observed between AMI patients and $\mathrm{PAD}$ patients (men: RR 9.62; 95\% CI 5.78 to 16.02, women: RR 14.28 ; $95 \%$ CI 6.68 to 30.52 ) In contrast, the 5 -year risk of death was similar for male AMI and PAD patients and differences in risks of death between AMI and ischaemic stroke patients largely attenuated.

Conclusions The dynamics of mortality over time clearly differ between atherosclerotic diseases. Short-term mortality is higher for cardiac and ischaemic stroke patients compared to PAD patients. However, the risk of death increases considerably over follow-up times for PAD patients, and 5 years after first hospital admission the differences in risks of death between AMI and PAD patients and between AMI and ischaemic stroke patients have largely attenuated.

\section{P54 MIGHT FINANCIAL CUTS DRIVE NHS DECISION-MAKING "DOWNSTREAM"? A OUALITATIVE STUDY OF FACTORS AFFECTING PUBLIC HEALTH DECISION-MAKING}

doi:10.1136/jech.2010.120477.54

L Orton, F Lloyd-Williams, D Taylor-Robinson, M O'Flaherty, S Capewell. Division of Public Health, School of Population Community and Behavioural Sciences, University of Liverpool, Liverpool, UK

Objectives To explore the process of public health decision-making, and the role of research evidence, taking cardiovascular disease (CVD) as a case study.

Design In-depth interview study.

Setting and Participants Over 30 public health policy-makers and planners in CVD, including: commissioners, public health consultants, data analysts, librarians and knowledge managers at Primary Care Trusts; public health academics; lead consultant cardiologists; local and national guideline developers; and third sector staff.

Methods In-depth semi-structured interviews were recorded and transcribed verbatim. Transcripts and field notes were analysed using the constant comparative method.

Findings Participants reported that previously there was relatively little change in health care investment year on year. Consequently, it was seldom necessary to seek research evidence. However, changes had occurred in recent times. National Health Service (NHS) cuts had led to a more systematic prioritisation process necessitating the explicit use of research evidence. There was a sense that decisionmakers must now take stock of what they were doing and ascertain if it was evidence-based. Unfortunately, these cuts had also removed much of the opportunity for creative thinking and for trying out new and unproven innovations. Despite most participants wishing 